\title{
Investigation of ferroresonanse phenomena and its possibilities of occurrence in the operation of 6-220 kV electromagnetic transformer
}

\author{
Andrew Tychkin ${ }^{1}$, Andrew Yablokov ${ }^{1}$, Vladimir Lebedev ${ }^{1}$, Nikita Rodin ${ }^{1}$, and Maksim Batmanov ${ }^{1}$ \\ ${ }^{1}$ Ivanovo State Power Engineering University, Ivanovo, Russia
}

\begin{abstract}
Ferroresonance phenomena lead to damage to electromagnetic measuring voltage transformers and nearby equipment. Damping devices and antiresonance transformers, which are used for preventing the occurrence of ferroresonance phenomena, are effective in a certain area of influencing factors. The article presents methods for studying the possibility of occurrence of ferroresonance phenomena in the operation of 6-220 kV voltage transformers based on physical and mathematical modeling. The results of the tests according to the developed method are the area of the existence of a dangerous ferroresonance for the voltage transformer under test or the confirmation of its antiresonance properties in a certain range of influencing factors under study. The article also describes an experimental setup based on RTDS for conducting experimental studies. The developed methods were tested in the studies of one of the types of antiresonance transformers.
\end{abstract}

\section{Introduction}

During the operation of electromagnetic voltage transformers (VT), ferroresonance phenomena [1-10] can occur, which serve as one of the reasons for their high damage. It is known that $6-10 \%$ of isolation monitoring voltage transformers installed in networks with an isolated neutral are damaged annually [11], while their average service life does not exceed 3-5 years [12]. Ferroresonance phenomena can be accompanied by fire and subsequent explosion of voltage transformers (Fig.1), which often leads to damage to nearby equipment and creates a dangerous situation for operating personnel.

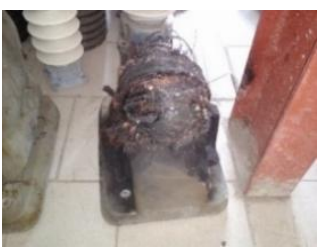

a)

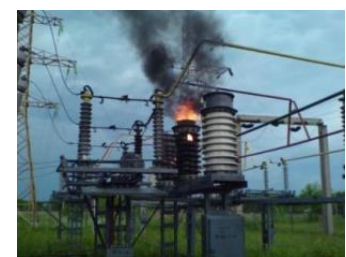

b)

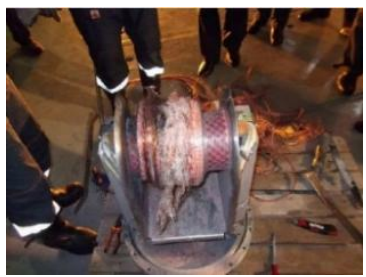

c)

Fig. 1. Voltage transformers damaged due to ferroresonance phenomena: a) ZNOL-35; b) NKF-110; c) Trench TFB sm 245.
In electric networks with an isolated neutral, ferroresonance can occur during intermittent arc singlephase earth faults (IASEF), the phenomenon of false earth, power line breakage, phase overturning [13-17]. In networks with a dead-earth neutral, these processes occur during switching and incomplete-phase modes of operation with power from a source with a capacitive resistance.

The possibility of occurrence of ferroresonance phenomena depends on many factors (voltage level, switching angle, network capacity, etc.). The areas of existence of dangerous ferroresonance for transformers of the NKF type are shown in Fig. 2 a). 


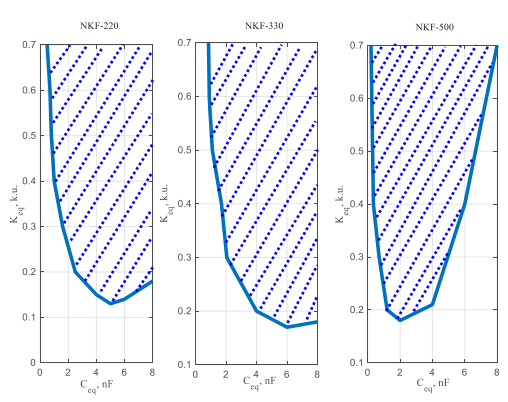

a)

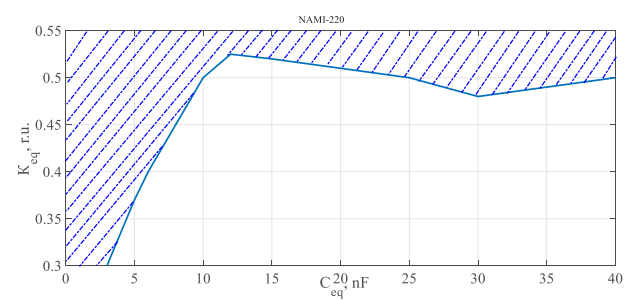

b)

Fig. 2. The region of existence of threat of ferroresonance for transformer type voltage NKF at the frequency of $50 \mathrm{~Hz}[18$, 19] (a) and NAMI-220 on the sub-harmonic $16.6 \mathrm{~Hz}$ [14] (b) where $\mathrm{K}_{\text {eq }}=\mathrm{CHVB}_{\mathrm{HV}} /\left(\mathrm{C}_{\mathrm{HVB}}+\mathrm{Cg}_{\mathrm{g}}\right), \mathrm{C}_{\mathrm{eq}}=\mathrm{C}_{\mathrm{HVB}}+\mathrm{C}_{\mathrm{g}}, \mathrm{C}_{\mathrm{HWB}}-$ total capacitance between open contacts of all breakers, $\mathrm{nF} ; \mathrm{C}_{\mathrm{g}}-$ total capacitance to ground of all elements of the disconnected part of switch-gear.

To prevent the possibility of occurrence of ferroresonance phenomena in networks are operated, the following measures are applied: resistive grounding of the neutral, connection of additional capacitances to the switched object; programming and disconnection of switching equipment [20-24].

In addition, special devices can be used to suppress ferroresonance phenomena, such as: ABB VT Guard Pro, Trench Ferroresonance Damping Device. They are installed on the secondary winding of the VT and when fixing a sharp increase in current, they increase the secondary resistance of the circuit (the process of damping). However, there are known cases of occurrence of ferroresonance when using such devices [17]. As an example, we can cite the accident at the 220 $\mathrm{kV}$ switch-gear of the Beskudnikovo substation at the end of the 2011 year, where, as a result of ferroresonance, the VT of the company Trench TVB sm 245 is damaged (Fig. 1, c)) [24].

In addition to special devices for protection against ferroresonance, there are antiresonance transformers of the NAMI, NALI NTZ type, etc. [20]. Antiresonance transformers do not guarantee complete protection against ferroresonance (fig. 1, c)) [20], [26]. At the same time, their cost is an order of magnitude higher than the cost of traditional VT. The performed studies [27] show that when we operate $110-500 \mathrm{kV}$, ferroresonance can occur only at $1 / 3$ of the subharmonic, with significantly lower current multiplicities compared to traditional VT, but when using traditional VT and VT of the NAMI type in parallel, the antiresonance properties of the latter sharply decrease, and ferroresonance can occur at a frequency of $50 \mathrm{~Hz}$.
The procedure for testing VT in order to assess the possibility of occurrence of ferroresonance is presented in Preliminary national standard (PNST 319-2018) [27] and is of the nature of a preliminary document. This standard requires improvements in terms of test methods, basic and structural test schemes, and the development of a list of recommended equipment for conducting these studies.

Thus, it is relevant to develop methods for studying ferroresonance phenomena and determining the areas of dangerous ferroresonance for voltage transformers of various types.

\section{Research methodology for ferroresonance phenomena in networks with isolated neutral}

The developed procedure for testing VT in order to assess the possibility of the occurrence of ferroresonance includes tests on an experimental setup, whose diagram is shown in Fig. 3, as well as studies based on simulation modeling.

The test setup uses a PONOVO 4Q four-quadrant amplifier (power $15 \mathrm{kVA}$ ) as a voltage source, controlled by software and hardware complex the Real Time Digital Simulator (RTDS). The four-quadrant amplifier is controlled via a fiber-optic communication line using the AURORA protocol. The output voltage of the fourquadrant amplifier is $270 \mathrm{~V}$, so specialized step-up transformers are used to increase the voltage to $6-35 \mathrm{kV}$. A precision digital combined measuring transformers (combined digital transformer) manufactured by LLC NGO «Digital Measurement Transformers» are used for measuring currents and voltages. Resistive dividers not subject to ferroresonant phenomena and not distort the voltage waveforms used as a primary voltage converter in these transformers. The network capacity is modeled by high-voltage oil-filled capacitors to ground. Simulation of arc intermittent single-phase earth faults is performed by specialized device. This device is controlled by the PONOVO PAV250Bi Voltage Amplifier. A potential difference is formed on two stationary spheres when voltage is applied with an amplitude of $5 \mathrm{~V}$. After that a third sphere starts to move, which moves from one sphere to another, forming an arc short circuit.

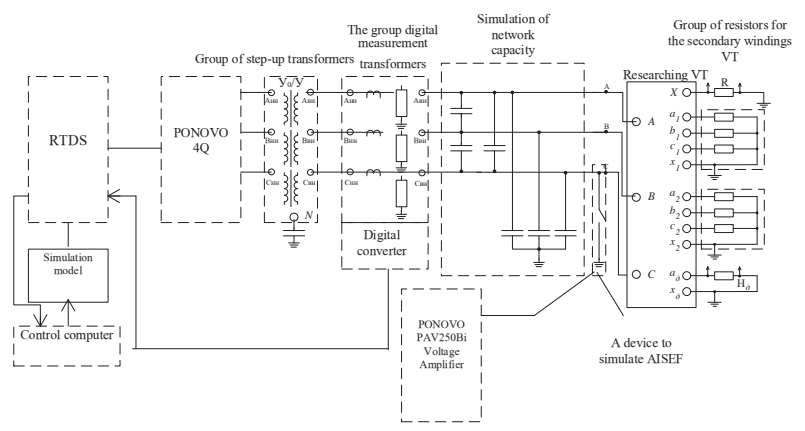

Fig. 3. Diagram of an experimental setup for conducting experiments to determine the resistance of a $6-35 \mathrm{kV}$ voltage transformer to ferroresonance phenomena. 
The described experimental setup allows the following tests to be carried out.

1. Investigation of the operation of the voltage transformer and the possibility of the occurrence of ferroresonance in the case of an alternating single-phase arc fault to earth. The highest operating voltage is applied to the transformer under test, and then a device that simulates an intermittent arc is turned on. The duration of the arc burning when closing is at least $1 \mathrm{~s}$, the number of breakdowns should be from 25 to 30 , the moment of closing is $0.8-0.9$ of the amplitude value, the number of experiments should be at least 20 .

2. Investigation of the operation of the voltage transformer and the possibility of the occurrence of ferroresonance when switching unloaded buses. The test consists in switching the voltage transformers on and off from the symmetrical source with isolated neutral at least ten times. The time difference between the closure of the switch contacts when closing is at least $5 \mathrm{~ms}$, and when disconnecting - at least $3 \mathrm{~ms}$, switching should be performed at a maximum voltage of $\pm 1 \mathrm{~ms}$.

3. Investigation of the operation of the voltage transformer and the possibility of the occurrence of ferroresonance when the network phase is inverted. The phase-to-earth capacitances are zero in this test. The reversal of the network phase is set programmatically in the hardwear and softwear suite RTDS.

During the tests, the following parameters of the tested transformer are monitored: voltages on high voltage windings, currents in high voltage windings, voltage at the terminals of the open triangle winding, current in the neutral of the primary windings, housing temperature.

Transformers are considered to have passed the test if the currents of their high-voltage windings, as well as the current in the neutral in all experiments, did not exceed the values of the thermal withstand currents of the rated duration, the temperature of the body of the transformer under test did not exceed the permissible value and the effective voltage value at the terminals of the open-delta winding did not exceed the natural voltage balance and no damage to the voltage transformer occurred.

The tests carried out on the described experimental setup have a number of disadvantages that are eliminated at the stage of research on simulation models.

1. The capacity of the network varies discretely with some step, and the maximum and minimum values are limited by the high-voltage capacitors available in the laboratory.

2. There is no possibility of reproducing the AISEF according to the three existing theories of their origin (Peterson, Peters-Slepyan, Belyakov).

3. Difficulty of performing a series of tests (for example, investigating the operation of a voltage transformer with a "false ground" phenomenon).

A voltage transformer performance study is carried out to develop a simulation model using the OMICRON VOTANO 100 tester. Parameters such as winding resistance, leakage reactance are measured, and the magnetization characteristic of the voltage transformer is also determined.
To conduct research, the following simulation models of electric power facilities are being developed in RTDS:

- a model of a ferroresonant circuit of an electrical network with an isolated neutral for investigating the operation of an electromagnetic voltage transformer with alternating arc single-phase earth faults (Fig. 4, a));

- a model of a ferroresonant circuit of an electrical network with an isolated neutral to study the operation of an electromagnetic voltage transformer in the event of a "false ground" phenomenon (Fig. 4, b));

- models of ferroresonant circuits of an electrical network with an insulated neutral for studying the operation of an electromagnetic voltage transformer in non-full-phase modes of operation (including, with partial-phase connections of network sections with an overhead power line and an unloaded power transformer) (Fig. 5).

In fig. 4 and fig. 5, the following designations are used: $R_{s}$ is the active resistance of the source, $L_{S}$ is the inductive resistance of the source, $\mathrm{R}_{\mathrm{S} 0}$ is the active resistance that simulates losses in the steel of the voltage source, $\mathrm{C}_{\mathrm{A}(\mathrm{B}, \mathrm{C})}$ is the equivalent capacitance of the phase to ground, $\mathrm{C}_{\mathrm{AB}}(\mathrm{BC}, \mathrm{CA})$ is the equivalent capacitance between phases, $\mathrm{R}_{\mathrm{lr}}$ - active leakage resistance, $\mathrm{R}_{\text {arc }}$ - arc resistance, $\mathrm{R}_{\mathrm{T}}$ - active transient resistance.

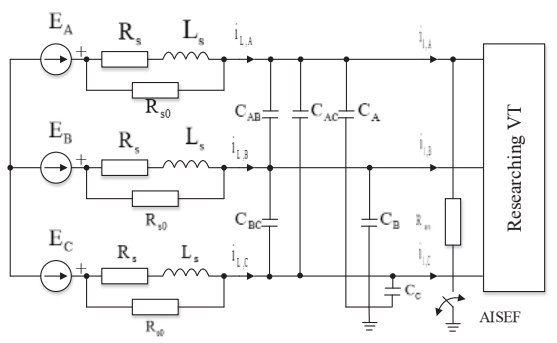

a)

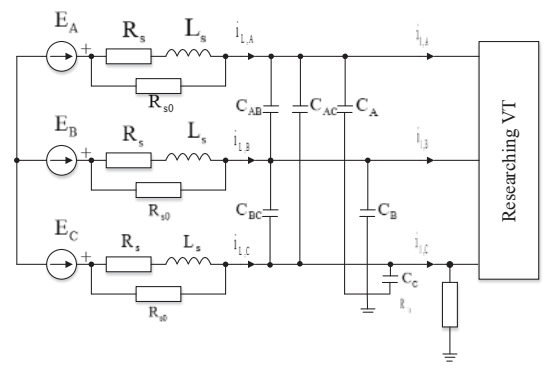

b)

Fig. 4. Model of a ferroresonant circuit of an electrical network with an isolated neutral for investigating the operation of an electromagnetic voltage transformer: a) with alternating arc single-phase earth faults; b) when the phenomenon of "false ground". 


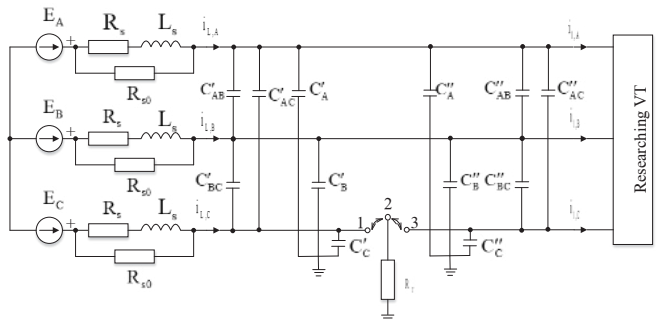

a)

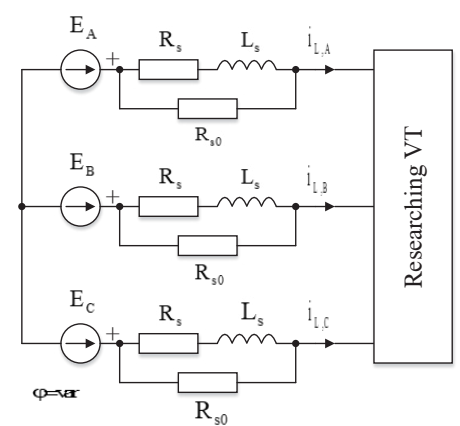

b)

Fig. 5. Models of ferroresonant circuits of an electrical network with an insulated neutral to study the operation of an electromagnetic voltage transformer in non-full-phase modes of operation: a) - an open overhead power line (in positions 1 an open line from the supply side; 2 - line do not touch the ground; 3 - an open line from the side TH); b - phase reversal.

The simulation model of the voltage transformer is described by the following equations [28]:

$$
\begin{gathered}
\frac{d \psi_{A}}{d t}=R_{0} \cdot\left(i_{1, A}+i_{2}^{\prime}-f\left(\psi_{A}\right)\right) \\
\frac{d \psi_{B}}{d t}=R_{0} \cdot\left(i_{1, B}+i_{2}^{\prime}-f\left(\psi_{B}\right)\right) \\
\frac{d \psi_{C}}{d t}=R_{0} \cdot\left(i_{1, C}+i_{2}^{\prime}-f\left(\psi_{C}\right)\right) \\
i_{1, A}=\frac{1}{L_{1}} \int\left(u_{A}-i_{1, A} \cdot\left(R_{1}+R_{0}\right)-R_{0} \cdot\left(i_{2}^{\prime}-f\left(\psi_{A}\right)\right)-u_{N}\right) d t \\
i_{1, B}=\frac{1}{L_{1}} \int\left(u_{B}-i_{1, B} \cdot\left(R_{1}+R_{0}\right)-R_{0} \cdot\left(i_{2}^{\prime}-f\left(\psi_{B}\right)\right)-u_{N}\right) d t \\
i_{1, C}=\frac{1}{L_{1}} \int\left(u_{C}-i_{1, C} \cdot\left(R_{1}+R_{0}\right)-R_{0} \cdot\left(i_{2}^{\prime}-f\left(\psi_{C}\right)\right)-u_{N}\right) d t \\
i_{2}^{\prime}=\frac{1}{3 \cdot L_{2}^{\prime}} \int\left(\left(-R_{0} \cdot\left(i_{N}+3 \cdot i_{2}^{\prime}-f\left(\psi_{A}\right)-f\left(\psi_{B}\right)-f\left(\psi_{C}\right)\right)-3 R_{2}^{\prime} \cdot i_{2}^{\prime}\right) d t\right. \\
u_{N}=R_{N} i_{N}+L_{N} \frac{d i_{N}}{d t} \\
i_{N}=i_{1 A}+i_{1 B}+i_{1 C}
\end{gathered}
$$

where $\mathrm{u}_{\mathrm{A}}, \mathrm{u}_{\mathrm{B}}, \mathrm{u}_{\mathrm{C}}$ - the instantaneous values of the phase voltage of the corresponding phases of the primary winding; $i_{1, A}, i_{1, B}, i_{1, C}$ - instantaneous value of the phase current of the primary winding of the voltage transformer; $i_{L, A}, i_{L, B}, i_{L, C}$ - instantaneous value of the line current of the EMF source. $\mathrm{R}_{1}$ - active resistance of the primary winding; $\mathrm{L}_{1}$ - inductance of the primary winding $\mathrm{u}_{\mathrm{N}}$ - neutral bias voltage; $\mathrm{i}_{2}$ - instantaneous value of the reduced current of the secondary winding; $\mathrm{R}_{2}{ }_{2}$ - reduced active resistance of the secondary winding; $\mathrm{R}_{2}^{\prime}$ - reduced inductance of the secondary winding; $\mathrm{R}_{0}$ active resistance in the magnetization branch, simulating losses in steel (for hysteresis and eddy currents); $\mathrm{f}\left(\psi_{\mathrm{A}}\right)$, $\mathrm{f}\left(\psi_{\mathrm{B}}\right), \mathrm{f}\left(\psi_{\mathrm{C}}\right)$ - the function of flux linkage change versus time of the corresponding phase; $\mathrm{R}_{\mathrm{N}}$ - active resistance of neutral; $\mathrm{L}_{\mathrm{N}}$ - neutral inductance; $\mathrm{i}_{\mathrm{N}}$ - the instantaneous value of the neutral current (depending on the design of the primary winding, if the connection is of the Y-0 type (with load), then the current is found according to the first Kirchhoff's law).

The verification of the developed simulation model of the voltage transformer in the RTDS is performed by comparing the results of experimental studies and studies on the simulation model under the same conditions.

On the developed simulation models in the RTDS, the following studies of the voltage transformer under test are carried out in order to determine the possibility of hazardous ferroresonance during its operation:

1. Investigation of the operation of the voltage transformer, the possibility of ferroresonance and overexcitation of the core in the case of an alternating single-phase arc fault to earth. Arc alternating singlephase earth faults should be modeled according to the theories of Petersen (Fig. 6, a), Peters and Slepyan (Fig. 6, b), Belyakov N.N. (Fig. 6, c), as well as asymmetric (the fault voltage on the positive and negative half-waves are different, the ratio is $1 / 0.8$ ) and non-periodic unipolar arcs. Different moments of insulation breakdown are set to simulate intermittent arcing earth faults according to different theories. Studies should be performed at different intensities of ignition and extinction of the arc (including at an intensity once per period or less).

According to research by a number of authors, traditional and anti-resonant voltage transformers are most often damaged due to the effect of an asymmetric or unipolar arc, as well as when the ignition and extinguishing of the arc occurs once in a period or less often (in the latter case, ferroresonance can be associated with the processes of charging and discharging the network capacity).

2. Investigation of the operation of the voltage transformer and the possibility of the occurrence of ferroresonance upon disconnection or self-elimination of a single-phase earth fault.

Ferroresonance is associated with the discharge of the zero sequence capacitance of the network through the grounded high voltage windings of the transformers in this mode. Ferroresonance is subharmonic and occurs at a frequency of $25 \mathrm{~Hz}$, according to the research of a number of authors in this mode.

3. Investigation of the operation of the voltage transformer and the possibility of the occurrence of ferroresonance when connecting the VT to the buses with a small total capacitance.

4. Investigation of the operation of the voltage transformer and the possibility of the occurrence of ferroresonance when switching unloaded buses. 
5. Investigation of the operation of the voltage transformer and the possibility of the occurrence of ferroresonance in incomplete-phase modes (when switching on and off a network section, power grid phase reversal).

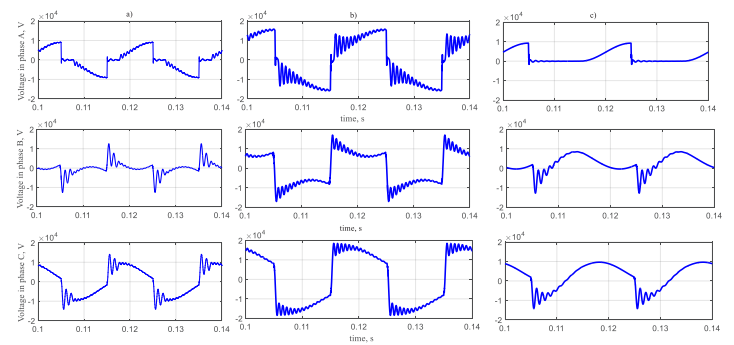

Fig. 6. Examples of voltage waveforms with an alternating single-phase arc-phase earth fault of phase A in a $6 \mathrm{kV}$ network, with a capacitive current of 24.2 according to theory: a) Belyakov; b) Petersen; c) Peters and Slepyan.

The developed technique was tested at the Scientific and Educational Center «High-voltage Digital Measuring Converters and Transformers » for testing VT in order to assess the possibility of the occurrence of ferroresonance. And based on the results of the research, it was decided to continue the cycle of finalizing the test methodology for voltage transformers in order to assess the occurrence of ferroresonance. The assembled experimental scheme is shown in Fig. 7.
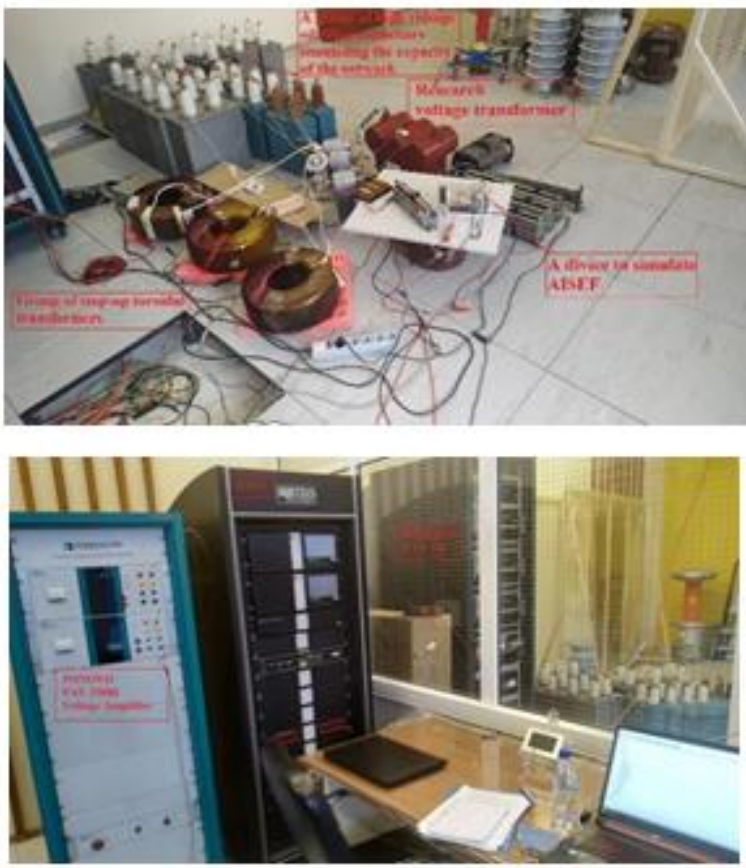

Fig. 7. Voltage transformer test circuit.

Oscillograms obtained as a result of experimental studies are shown in Fig. 8 and Fig. 9.

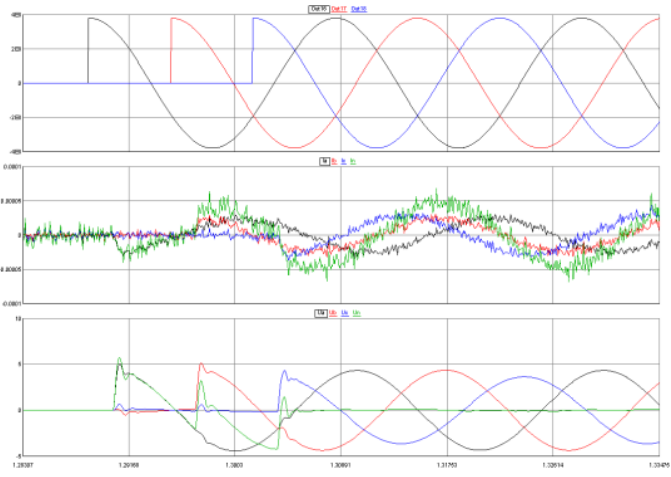

a)

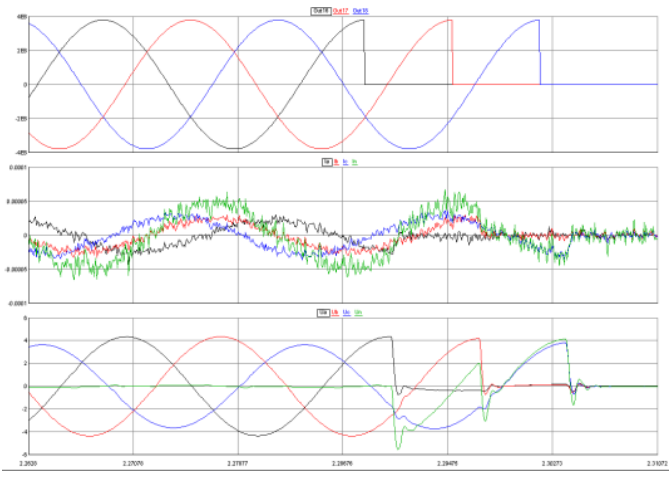

b)

Fig. 8. Testing the voltage transformer and assessing the possibility of ferroresonance when switching on unloaded buses at rated voltage from the high voltage side a) sequential connection of phases; b) sequential disconnection of phases.

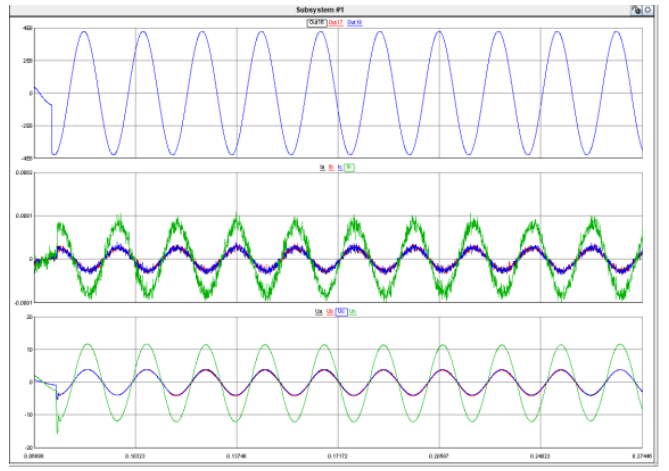

a)

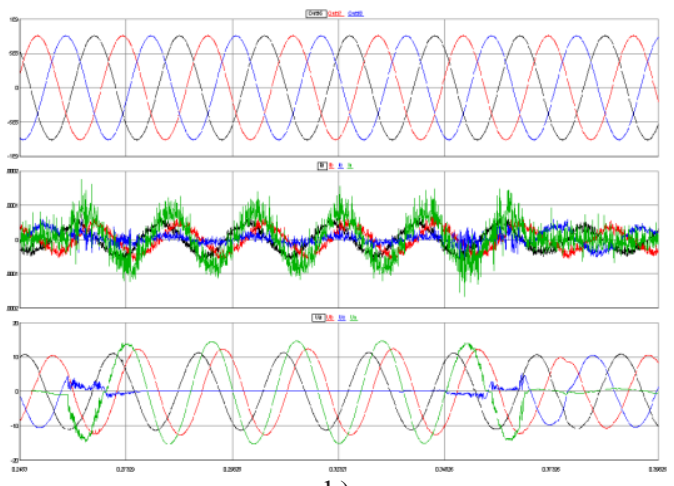

b)

Fig. 9. Testing the voltage transformer and assessing the possibility of ferroresonance: a) "phase reversal" at rated voltage from the high voltage side; b) intermittent arc singlephase earth fault at rated voltage on the high voltage. 
The results of testing according to the developed technique are the area of existence of dangerous ferroresonance for the voltage transformer under test or confirmation of its antiresonant properties in a certain investigated range of network capacities.

\section{Method of investigation of ferroresonance phenomena in networks with a grounded neutral}

Conducting experimental studies of the possibility of the occurrence of ferroresonance during the operation of transformers of voltage $110 \mathrm{kV}$ and higher at existing power facilities or special landfills is associated with significant time and money resources. The scheme of the experimental setup for these studies is shown in Fig. 10 $[18,27]$. However, according to [27], tests on the open distribution-220 $\mathrm{kV}$ and higher can be replaced by simulation modeling, when using approved models.

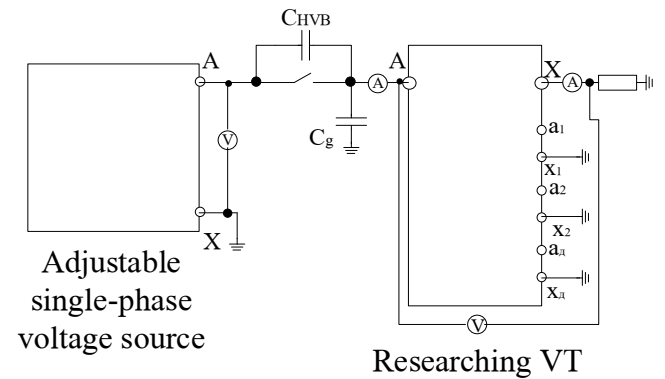

Fig. 10. Test circuit of a voltage transformer for networks with a grounded neutral of the voltage class $220 \mathrm{kV}$ and higher.

A simulation model was created and tested [29, 30], described by the following equations for studying the ferroresonance phenomena in networks with a grounded neutral.

$$
\begin{gathered}
e=E_{m} \sin (\omega t+\phi) \\
\frac{d i}{d t}=\frac{\frac{d \psi}{d t}}{\frac{d \psi}{d i}} \\
\frac{d \psi}{d i}=\frac{d(A \cdot \operatorname{arctg}(B \cdot i)+C \cdot i)}{d i}=\frac{A \cdot B}{1+(B \cdot i)^{2}} \\
\frac{d u_{C H V B}}{d t}=\frac{i-C_{H V B} \cdot E_{m} \cdot \omega \cdot \cos \left(\omega t+\frac{\pi}{2}\right)}{C_{H V B}+C_{g}} \\
u=e-u_{C H V B}
\end{gathered}
$$

where $\mathrm{e}$ - the instantaneous value of the EMF; $E_{m}$ - the amplitude of the EMF; $\varphi$ - the phase of the switching time; $\mathrm{i}$ - the instantaneous current in the primary winding VT, $\psi$ - the flux linkage produced in the windings $\mathrm{VT} ; \mathrm{R}$ - the equivalent resistance of the primary winding $\mathrm{VT}$; $\mathrm{u}_{\mathrm{CHWS}}$ - the instantaneous voltage between open contacts of all of the switches; A, B, C are the coefficients of the approximation of the magnetization curve; $\mathrm{C}_{\mathrm{HWB}}$ - total capacitance between open contacts of all circuit breakers, $C_{g}$ - the total capacity of the equipment relative to the ground; $\mathrm{u}-$ the instantaneous voltage at VT.

Based on the developed simulation model, studies were carried out for the NKF-220 transformer (the most common type of VT in the Russian Federation), and for the transformer with an open magnetic core (one of the possible designs that reduce the possibility of the occurrence and development of ferroresonance). The results of the conducted studies (Fig. 11) showed the effectiveness of using voltage transformers with an open magnetic circuit in comparison with traditional VT of the NKF-220 type. The ratio of the zones of dangerous and safe ferroresonance in a transformer with an open magnetic circuit is much lower.

It should be noted that the use of antiresonance voltage transformers with an open magnetic circuit or NAMI-220 does not provide complete protection against the occurrence of ferroresonance, but it significantly reduces both the possibility of occurrence of ferroresonance phenomena and the level of currents in the transformer windings.

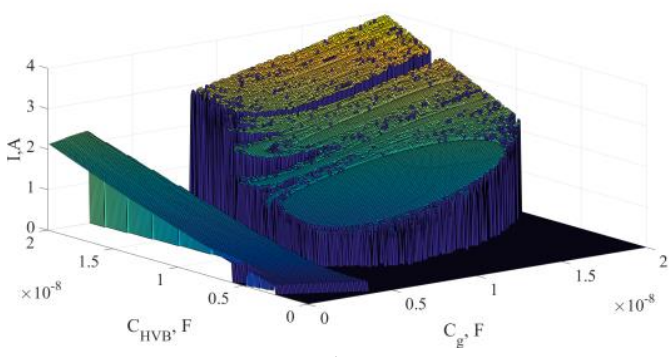

a)

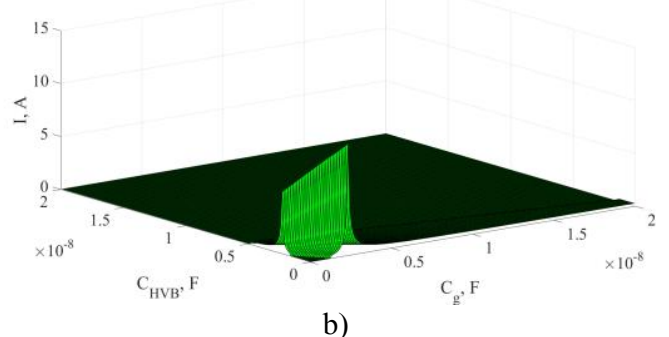

Fig. 11. Bifurcation diagrams at a switching angle of 0 degrees for NKF-220 (a) transformers and a voltage transformer with an open magnetic circuit (b).

\section{Conclusion}

The methods presented in the article allow testing antiresonance and traditional designs of 6-220 kV voltage transformers in order to determine the possibility of occurrence of ferroresonance phenomena during their operation. The developed methods were tested in the Scientific and Educational Center "High-voltage Digital Measuring Converters and Transformers". It should be 
noted that the phenomenon of ferroresonance is not fully understood and requires further research. While the equipment that is affected by ferroresonance is in operation, it is necessary to know the safe zone in which this equipment can operate steadily.

\section{References}

1. Rao Jwala Laxmi Narasimha, Bansal Gaurav, Auddy Soubnik, Tulkiewicz Thomas, Ohrstrom Magnus, Mitigation of ferroresonance in line commutated, HVDC converter interconnected with series compensated overhead line transmission system, India, Sweden (2016)

2. Shabain Heba Abu, Osman Ahmed, El-Hag Ayman, Detection and identification of ferroresonance, United Arab Emirates (2017)

3. Rezaei Salman, Impact of transmission line and plant outage on ferroresonance in AC transmission system and new suppression method, Iran (2017)

4. S. Poornina, Dr.C. Pugazhendhi Sugumaran, Identification of ferroresonance phenomena using, Wavelet transforms (India, 2016)

5. H.E. Zhiqiang, L.I. Xin, Q.I.N. Jiayuan, H. Haibo, Study on ferroresonance over-voltage based on harmonic elimination device, China (2018)

6. Wahyudi Mochammad, Negara I Made Yulistya, Asfani Dimas Anton, Hernanda I Gusti Ngurah Satriyadi, Hidayat Reno, Comparison of ferroresonance response on three phase transformer with different core material: M5 and ZDKH, Indonesia (2018)

7. Wahyudi Mochammad, Negara I Made Yulistya, Asfani Dimas Anton, Hernanda I Gusti Ngurah Satriyadi, Anugrah Kadek Suparta, Study of Peterson coil grounding system inductance variation on ferroresonance in $150 \mathrm{kV}$ transformer, Indonesia (2018)

8. Wahyudi Mochammad, Negara I Made Yulistya, Asfani Dimas Anton, Hernanda I Gusti Ngurah Satriyadi, Anugrah Bonifaccius Kevin Yegar Sahaduta, Study of ferroresonance in $150 \mathrm{kV}$ high voltage inductive voltage transformer, Indonesia (2020)

9. Wang Yunfei, Liang Xiaodong, Pordanjani Iraq Rahmi, Jafari Ali, Cui Ryan, Clark Colin, Investigation of ferroresonance causing sustained high voltage at a de-energized $138 \mathrm{kV}$ Bus: A case study, Canada (2019)

10. Solak Krysztof, Rebizant Waldemar, Modeling of ferroresonance phenomena in MV Networks, Poland (2018)

11. A.K. Popov, Investigation of ferroresonance overvoltages in 6-35 kV networks with isolated and resonantly grounded neutral, SaintPetersburg, 3 (2018)
12. A.V. Makarov, On the efficiency of functioning of antiresonance voltage transformers, Energoexpert, 6 (2013)

13. Wahyudi Mochammad, Negara I Made Yulistya, Asfani Dimas Anton, Hernanda I Gusti Ngurah Satriyadi, Fahmi Daniar, Application of Wavelet Cumulative Energy and Artificial Neural Network for Classification of Ferroresonance Signal During Symmetrical and Unsymmetrical Switching of Three-Phases Distribution Transformer, Indonesia (2017)

14. L. Jiaxin, L. Xuchen, W. Yanan, W. Defu, T. Jianeng, Discriminate Method of Power Frequency Ferroresonance in System with NonEffectively Earthed Neutral of Three-Phase Enclosed GIS, China (2018)

15. S. Boutora, H. Bentarzi, Ferroresonance study using false trip root cause analysis, Algeria (2019)

16. S.K. Chakravathy, C.V. Nayar, Series ferroresonance in power system (1995)

17. Zulkumarnain Abdul-Malek, Kamyar Mehranzamir, Benham Salimi, Hadi Nabipour Afrouzi, Saeed Vahabi Mashak Investigation of ferroresonance mitigation techniques in voltage transformer using ATP-EMTP simulation, Indonesia (2013)

18. Organization Standard Rosseti FGC UES, Guidelines for protection against resonant voltage increases in electrical installations of $6-750 \mathrm{kV}$ (2014)

19. Procedural Guidelines for the Prevention of Ferroresonance in 110-500 kV Switchgear with Electromagnetic Voltage Transformers and Switches Containing Capacitive Voltage Dividers, USSR Minenergo (1987)

20. O.I. Laptev, Research of efficiency of antiresonance voltage transformers of NAMI type in electric networks of high and medium voltage

21. Radmanesh Hamid, Distribution network protection using smart dual functional series resonance-based fault current and ferroresonance overvoltage limiter, Iran (2018)

22. Radmanesh Hamid, Heidary Amir, Smart solidstate ferroresonance limiter for voltage transformer application: principle and test, Iran (2018)

23. Shijin Tian, Tianlong Zhang, Xuezhong Liu, Hongwen Lui, Ruigui Li, Numerical and experimental simulation researches on characteristics of ferroresonance based on distributed power grid parameters, China (2016)

24. I.G.N. Satriyadi, Negara I Made Yulistya, Daniar Fahmi, Dimas Asfani Anton, Satria S. Simamora, Study om damping reactor installation to address ferroresonance on incoming $20 \mathrm{kV}$ GIS, Indonesia (2016) 
25. K.E. Kornilov, A.V. Makarov, Ferroresonance phenomena in electrical networks

26. K.P. Kadomskaya, O.I. Laptev, Anti-resonant voltage transformer, The effectiveness of the application, Electrical Engineering News, 6 (42), (2006)

27. Preliminary national standard of the Russian Federation, PNST 319-018, Measuring transformers part 3, Technical specifications for inductive voltage transformers, Moscow (2018)

28. O.I. Laptev, Investigation of the efficiency of antiresonance voltage transformers of the NAMI type in 6-35 kV networks with an isolated neutral, Novosibirsk

29. A.A. Yablokov, Development and research of the primary converterthe voltage of the measuring transformer fordigital substation 110-220, Ivanovo (2016)

30. N.A. Antonov, Analysis of ferroresonance circuits of $110-500 \mathrm{kV}$ electrical networks by mathematical modeling methods, Ivanovo (1998) 